\title{
Philosophiques
}

\section{Les explications causales en histoire}

\section{W. H. Dray}

Volume 4, numéro 1, avril 1977

URI : https://id.erudit.org/iderudit/203061ar

DOI : https://doi.org/10.7202/203061ar

Aller au sommaire du numéro

Éditeur(s)

Société de philosophie du Québec

ISSN

0316-2923 (imprimé)

1492-1391 (numérique)

Découvrir la revue

Citer cet article

Dray, W. H. (1977). Les explications causales en histoire. Philosophiques, 4(1),

3-34. https://doi.org/10.7202/203061ar

Ce document est protégé par la loi sur le droit d'auteur. L'utilisation des services d’Érudit (y compris la reproduction) est assujettie à sa politique d'utilisation que vous pouvez consulter en ligne.

https://apropos.erudit.org/fr/usagers/politique-dutilisation/ 


\title{
LES EXPLICATIONS CAUSALES EN HISTOIRE
}

\author{
par W. H. Dray
}

Le titre donné à cet écrit ne rend pas tout à fait justice au sujet de mon exposé.' Car ce dont je voudrais vous entretenir dépasse ce qu'on entend habituellement par "explication causale en histoire". Ce sur quoi je voudrais attirer votre attention en effet, ce sont trois aspects généraux qui caractérisent une grande partie de l'explication historique. Il s'agit de trois traits relatifs au type de compréhension que les historiens visent ordinairement à réaliser lorsqu'ils s'adonnent à leur oeuvre de reconstruction des événements et des états de choses du passé. La notion de cause se trouve liée de différentes façons à ces trois aspects, comme j'essaierai de le montrer dans la suite. Toutefois je ne me limiterai point aux explications qu'on trouve habituellement exprimées dans un langage explicitement causal.

Encore en guise de préliminaire, une seconde remarque s'impose. C'est qu'en affirmant que la compréhension historique comporte les trois aspects que je vais discuter je n'ai nullement le sentiment de faire oeuvre bien originale. Ces trois aspects furent amplement étudiés récemment par des philosophes de l'histoire de la tradition analytique. De fait, $j$ 'ai l'intention de profiter de cette occasion pour réfléchir sur leurs doctrines. D’une part, je vous offrirai une révision critique des propos tenus par certains de ces philosophes sur le sujet ; d'autre part, j'établirai aussi clairement que possible ce qui m'apparaît à la fois vrai et important dans leurs dires (j'oserai même les interpréter quelque peu à l'occasion). Pour situer leurs prétentions dans une juste perspective, il conviendra de les mettre en regard d'une conception de la compréhension historique qui eut l'heur de déclencher et de stimuler dans une large

1. Cet article a été écrit pour le Séminaire d'épistémologie et d'histoire des sciences, II, à l'Université du Québec à Montréal. Je voudrais remercier mes collègues de l'Université d'Ottawa, surtout les professeurs B. Garceau, J. Croteau et Y. Lafrance, pour l'aide qu'ils ont apportée dans sa préparation. 
mesure la philosophie analytique de l'histoire au cours des trois dernières décennies. J'ai l'espoir en ce faisant de fournir, particulièrement à ceux qui sont de trad un philosophique assez différente, une sorte d'échantillonnage de la façon typique de faire de la philosophie chez ces auteurs - façon caractérisée par un espèce d'analyse conceptuelle.

La théorie que je veux employer comme point de référence fait dépendre la compréhension historique - et, en vérité, la compréhension en général - de la découverte d'une explication, appelée " explication par une loi de couverture " (covering law explanation*), de ce qui doit être compris. Cette conception est devenue classique à la suite de sa présentation dans un article célèbre de C.G. Hempel publié en 1942 et intitulé "The Function of General Laws in History ${ }^{2}{ }^{2}$ On pourrait formuler les principes fondamentaux de la thèse de Hempel comme suit. Comprendre l'occurrence d'un événement, c'est être capable de répondre sur une base empirique à la question : Pourquoi en fut-il ainsi ? Ceci exige la connaissance des conditions antécédentes qu'on appelle ordinairement ses causes. Or la question cruciale est de savoir de quel droit nous pouvons représenter un événement subséquent comme faisant suite nécessairement à certaines conditions antécédentes. Hempel, pour sa part, prétend que nous ne sommes justifiés de le faire que dans le cas où il y a une loi générale, ou bien des lois générales, qui impliquent une telle succession. Seules de telles lois, constate-t-il, peuvent conférer une valeur explicative à un ensemble de conditions antécédentes.

Pour parler d'une façon plus formelle : expliquer un événement, c'est montrer que l'énoncé établissant son occurrence se déduit rigoureusement de prémisses qui affirment des lois ainsi que des événements antécédents. De là est né le terme "nomologicodéductif ", lequel est aussi employé pour désigner cette théorie de l'explication. À noter que Hempel n'a pas effectivement démontré pourquoi une explication doit répondre aux sévères exigences d'une structure strictement déductive. Il a présenté sa thèse comme chose intuitivement évidente, tout en ajoutant - comme si c'était une

* Cette traduction de " covering law " par "loi de couverture " est celle de Monsieur Bertrand Saint-Sernin dans Éléments d'épistémologie de Carl G. Hempel (Armand Colin, 1972, p. 79). Ce volume est la traduction de Pbilosophy of Natural Science (Prentice-Hall, 1966).

2. Journal of Philosopby, XXXIX, 1942, et dans H. Feigl et W. Sellars (éditeurs), Readings in Philosophical Analysis, Appleton-Century-Crofts, 1949, 469-471. 
raison suffisante - que ce qu'il préconisait était monnaie courante en science physique. D'autres pourtant ont suggéré des raisons pour justifier cette position, et celles-ci se ramènent pour la plupart à la suivante : si ce qu'on offre comme explication de l'occurrence d'un événement n'exclut pas sa non-occurrence, on ne comprend vraiment pas pourquoi, en ce cas, l'événement a eu lieu, plutôt que le contraire. Le seul moyen d'exclure une non-occurrence est assurément de montrer qu'une occurrence est strictement déductible. Et le seul moyen de faire une telle déduction, selon l'argument, est de recourir aux lois générales.

Évidemment, si on accepte ces critères de l'explication, la recherche historique se trouve dans de bien mauvais draps. On doit remarquer en effet que les historiens, quand ils prétendent donner des explications, non seulement offrent rarement des arguments explicitement déductifs, mais de plus ne pourraient pas souvent les formuler, même s'ils voulaient le faire. Quelle conclusion devaiton en tirer? Peut-être que l'histoire n'est pas véritablement une discipline explicative, et qu'elle ne fournit donc aucune compréhension du passé - et cela en dépit de l'usage fréquent que les historiens feront de termes tels que "parce que ", "donc", "par conséquent "? La conclusion à laquelle Hempel lui-même est arrivée est heureusement un peu plus modérée.

Tout d'abord, il prétend que, bien que le concept de l'explication qui sous-tend toute histoire respectable doive se conformer au modèle "nomologico-déductif ", ce qu'offrent les historiens pourrait être admis comme une " approche " d'explication, une explication "incomplète ", ou une "esquisse " d'explication. Hempel, néanmoins, insiste sur le fait qu'une bonne esquisse, tout en ne mentionnant aucune loi explicative, doit au moins suggérer la direction dans laquelle de telles lois devraient être recherchées. Mais il reconnaît finalement que même cette exigence est trop rigide. Car les lois qui pourraient être le plus clairement suggérées par une soi-disant explication historique incomplète sont celles qui généralisent la connexion particulière qu'on aura affirmée entre l'antécédent et le conséquent. Or une loi dérivée de cette façon est presque toujours plus contestable en soi que l'explication qui est dite dépendre d'elle. Ainsi - pour ne prendre qu'un des exemples donnés par $\mathrm{Hempel}^{3}$ - expliquer la migration des fermiers de

3. Op. cit. 464 . 
Dust Bowl vers la Californie par leur désir d'échapper à la sécheresse et aux tempêtes de sable, est beaucoup plus admissible que d'affirmer que de tels désirs sont suivis régulièrement par de telles migrations. Et si de telles explications, en quelque sorte, suggèrent des lois plus compliquées et mieux fondées, Hempel est forcé de concéder que, dans des cas typiques, celles-ci restent inconnues des historiens.

Il est dès lors peu surprenant de voir Hempel s'avancer audelà de sa première réponse au problème vers une seconde solution plus radicale. Le modèle "nomologico-déductif " lui-même, concède-t-il finalement, a besoin de quelque modification. Car il suffit pour qu'il y ait explication véritable, et non seulement simple approximation d'explication, de démontrer que les conditions antécédentes auront été telles qu'elles rendent le résultat donné plus probable. Pour montrer cela il faudra sans doute se référer à des lois. Mais les lois dans ce cas n'auront plus à être universelles ou sans exceptions. Du même élan, faudra-t-il abandonner la règle exigeant qu'on puisse déduire rigoureusement ce qui doit être expliqué. En effet, Hempel ajoute à sa théorie originale un second modèle d'explication, un modèle "nomologico-inductif ", qui emploie typiquement des lois d'ordre statistique. Et il fait remarquer - plutôt tardivement - que l'explication prend communément cette forme moins rigoureuse, même dans les sciences naturelles.

Je n'ai guère l'intention de m'engager dans un exposé détaillé de la théorie hempelienne. Un point cependant mérite particulièrement d'être signalé. Il a trait au passage de la version déductive du modèle à la version inductive. Ce passage est accompli chez Hempel sans aucune justification théorique quelconque. De plus, c'est une démarche qui est absolument exclue par l'argument général à l'appui de la théorie de la loi de couverture (" covering law ") que j'ai abordée il y a un moment, car elle admet qu'un événement puisse être explicable sans avoir eu lieu de façon nécessaire. Il est vrai que Hempel lui-même ne s'est pas servi de l'argument cité. Peut-être prétendrait-il que sa position finale se justifie au contraire du fait qu'on admette les deux formes d'explication dans les sciences naturelles. Cependant, dans l'exposé de Hempel, cette dernière considération se présente comme une réflexion supplémentaire ; et il est suffisamment clair que l'option inductive fut 
élaborée effectivement en réponse à un problème tout à fait différent. C'est que, si l'explication "nomologico-déductive " est le seul type légitime, rien ou à peu près rien en histoire n'aurait jamais été expliqué. Or c'est là une conclusion à laquelle Hempel ne voulait pas être forcé d'aboutir.

En d'autres termes, le changement de doctrine est marqué d'un certain opportunisme. Il est régi par des considérations d'ordre pragmatique, et non théorique. Hempel ne veut pas, apparemment, que l'analyse philosophique d'une discipline actuelle s'éloigne de la pratique de cette discipline, au point que ses conclusions ne lui soient plus applicables. Je n'ai aucune objection, pour ma part, à la révision de Hempel en tant qu'expression d'un tel intérêt. En effet, je serais content de m'en servir comme d'un précédent pour l'acceptation d'autres déviations de son modèle original si d'autres considérations pratiques les justifiaient. Mais il est important d'expliquer un peu plus clairement ce que je veux dire par là. Je ne suggère pas que tout ce qu'il arrive à un historien d'appeler une explication devrait être accepté comme telle par un philosophe, abstraction faite de sa structure ou de sa forme logique. Évidemment il y a de la bonne et de la mauvaise histoire. Mais lorsque ce qui est intuitivement accepté comme explication dans un certain genre d'enquête ne peut pas répondre effectivement aux exigences d'un modèle logique donné, - particulièrement s'il s'agit d'un modèle conçu a priori - il reste au philosophe autre chose à faire que de déclarer cette explication déficiente ou incomplète. Car il se peut qu'elle soit comme telle complète, mais d'un genre jusque-là inconnu. En d'autres termes, elle pourrait être régie par un concept d'explication tout à fait différent. Et l'une des raisons principales pour laquelle les philosophes d'une discipline ne sauraient se permettre d'en ignorer la pratique est que, s'ils le font, ils risquent d'en fausser les concepts fondamentaux.

Peut-être y a-t-il lieu d'interpréter la progression de Hempel du premier au second modèle comme étant plus qu'une réponse à la pratique en termes de simple expédient. Peut-être faudrait-il la voir comme une réponse plutôt au niveau de la théorie conceptuelle - disons, comme une reconnaissance du fait que l'explication et la compréhension en histoire peuvent s'établir dans la perception de la probabilité tout autant que de la nécessité. Mais s'il en est ainsi, 
il serait important de noter que le second modèle de Hempel est bien loin d'épuiser les possibilités d'une telle réponse au niveau théorique. Les philosophes dont je voudrais maintenant examiner les doctrines ont tous d'autres options à proposer.

Mon premier philosophe non hempelien est Alan Donagan de l'Université de Chicago. Donagan a apporté de nombreuses contributions importantes à la littérature philosophique au chapitre de l'histoire, et ses vues n'ont pas manqué d'évoluer. Je vais examiner d'abord la critique qu'il fit de la théorie hempelienne en 1957 dans un article intitulé "Explanation in History ». ${ }^{4}$ Puis je lierai la thèse de cet article à quelques-unes de ses doctrines un peu plus récentes à propos du même problème.

Quand, au départ, Donagan s'avise de mettre à l'épreuve les vues de Hempel, tout en tenant compte de ce que les historiens admettent intuitivement comme explication, il trouve son modèle souvent inapplicable, même sous forme inductive. Voici un des exemples qu'il considère - il s'agit d'une explication tirée d'un récit de l'historien anglais, J.A. Williamson.' Ce qui demande une explication, en ce cas, est le fait que les Danois, qui parcouraient les côtes de l'Angleterre pendant les premières décennies du neuvième siècle, bien qu'ils aient eu le pouvoir de s'y installer et que la terre fût bonne, n'ont pas saisi l'occasion de le faire. L'explication de Williamson est, pour le dire brièvement, que les Danois étaient d'abord et avant tout des pillards, et ensuite, mais ensuite seulement, des colons. En effet, il y avait ailleurs une abondance de butin à leur portée. Donc ils ont mis à sac leurs conquêtes, puis ont poursuivi leur chemin.

Or se demande Donagan, quelle peut bien être la loi générale en cause dans une pareille explication? Aucune, répond-il, même si on insiste pour que l'explication soit déductive. Telle quelle, elle contient certes une affirmation générale : elle attribue une caractéristique générale aux Danois du neuvième siècle. Et de cela nous pourrions peut-être déduire ce que ces agents feraient dans les cir-

4. Mind, 1957, et dans P. Gardiner (éditeur), Theories of History, Free Press, 1959, 428-443

5. Op. cit. 432. 
constances envisagées, en ajoutant des prémisses concernant leurs croyances et les occasions qui leur étaient offertes. Mais la simple affirmation que les Danois étaient avant tout des pillards n'est pas une loi, soit psychologique, soit sociologique, ou même historique. Ce n'est pas une loi du tout, puisqu'elle n'affirme rien sur la façon dont un certain type de personne ou de groupe se comporte. Ce qu'elle affirme n'a trait qu'à la manière dont les Danois se comportent, c'est-à-dire au comportement particulier d'un peuple historiquement identifiable, un individu historique collectif. Cette situation est logiquement parallèle à celle où l'on explique les actes d'une personne particulière, non point à l'aide d'une théorie de la conduite d'une personne de son type, mais en fonction de ses idiosyncrasies personnelles.

On devrait remarquer que, si ces prétentions de Donagan sont correctes, la théorie philosophique touche ici à des manières de parler très familières aux historiens - surtout quand ils s'opposent à un type de pression méthodologique qu'on soupçonne souvent découvrir dans une approche comme celle de Hempel. L'histoire, entend-on dire souvent, s'intéresse à l'unique et au particulier. L'historien doit donc chercher à comprendre, non pas par appel aux théories générales - théories empruntées peut-être aux sciences sociales générales - mais en s'immergeant, pour ainsi dire, dans la période ou le développement envisagés, en y apprenant à les connaître en leurs particularités concrètes. Or en prétendant que le seul genre de connaissance générale qui soit exigé logiquement par (au moins) un type d'explication des actions en histoire se constitue lui-même par des faits historiques, Donagan fournit une base théorique à cette façon de parler. Il valorise aussi des slogans comme celui de l'historien-philosophe, R.G. Collingwwod, ${ }^{\circ}$ affirmant que l'histoire est, en un sens non négligeable, une discipline autonome.

Mais cette théorie de Donagan pourrait-elle survivre à la critique qu'on lui fera assurément? Par exemple, pourrait-on vraiment déduire ce que des individus ou des groupes feront à partir de la connaissance de leurs caractères et de leurs circonstances? Donagan doit répondre à cette critique parce qu'il accepte l'argument déductiviste a priori que j'ai indiqué il y a un moment. Ce qu'il nie est que, pour être déductive, une explication ait toujours

6. The Idea of History, Clarendon Press, 1946, V. 
besoin de lois générales. Selon lui, en certains cas, des affirmations de traits de caractère suffisent. Mais les concepts qu'on emploie dans de telles "explications par le caractère " sont - pour parler comme F. Waismann - "logiquement poreux ".' Ainsi, du seul fait que les Danois étaient avant tout des pillards, on ne saurait conclure rigoureusement qu'ils pilleraient dans toutes les circonstances appropriées. Pour être acceptable, l'explication n'en demande pas tant. Il lui suffit qu'ils agissent ainsi la plupart du temps. Cette "porosité " du concept, bien entendu, ne poserait aucun problème si Donagan était prêt à élaborer, comme Hempel l'a fait, et pour des raisons aussi pragmatiques, une version plus faible et non déductive de son propre modèle - une version probabiliste. Et, bien que Donagan s'y oppose, c'est là une modification que volontiers j'admettrais moi-même. Les historiens me surprennent néanmoins de temps en temps par la facilité avec laquelle ils se montrent disposés apparemment à adopter l'idéal déductif, au moins dans leurs moments de théorisation. Geoffrey Elton, par exemple - un historien constitutionel anglais, assez conventionnel bien que très éminent - propose, dans son livre The Practice of History, et cela presque comme critère de la compréhension historique, que l'historien se familiarise avec son sujet au point qu'il puisse deviner ce que par la suite ses sources auront à lui révéler. ${ }^{8}$ Ce que Donagan voudrait ajouter, c'est que si jamais un historien se trouvait dans une situation aussi enviable, ce ne serait pas nécessairement à cause de sa connaissance de lois générales.

Une critique de la théorie de Donagan qui paraîtra peut-être un peu plus provocante théoriquement est la suivante. Même si une explication par référence au caractère individuel ne mentionne aucune loi, n'est-il pas vrai que quelques lois seront présupposées ou impliquées par le caractère dont elle affirme l'existence? On entend dire quelquefois, par exemple, qu'on ne peut pas soutenir empiriquement une telle affirmation sauf par appel finalement aux lois générales. Et on considère quelquefois comme pertinente à ce sujet une comparaison avec des explications similaires d'événements naturels. Voici un exemple qu'a donné Gilbert Ryle et que

\footnotetext{
7. Donagan, op. cit., 436.

8. Fontana, 1969, 30. Cité par Mink dans "History and Fiction as Modes of Comprehension ", New Literary History, I, 1970.
} 
Donagan lui-même examine ${ }^{9}$. Quand une vitrine est cassée par une pierre, l'éclatement peut être expliqué, disons, par la fragilité de la glace. Mais si nous savons qu'un carreau particulier est fragile, c'est sûrement parce que nous croyons avoir affaire à une qualité dispositionnelle de toute glace. Ryle soutient néanmoins, sur un plan strictement logique, qu'on pourrait apprendre les dispositions des choses individuelles et ignorer en même temps les lois générales auxquelles elles sont effectivement soumises. Donagan est d'accord ; et je le suis aussi. Si cela ne saute pas immédiatement aux yeux dans le cas que nous venons de considérer, la raison en est, sans doute, que les vitrines ne sont justement pas le genre de chose dont nous découvrons habituellement les qualités en les examinant individuellement. Il ne nous viendrait pas à l'esprit de lancer des pierres dans une glace particulière pour voir si elle aussi est fragile. Par ailleurs, nous pourrions apprendre si une porte donnée est bruyante ou non, en essayant de la mouvoir, et même plus probablement - si tel ou tel homme est jaloux ou vindicatif en l'exposant à la tentation.

Toutefois il reste vrai que, dans le cas des événements d'ordre physique, une explication en termes des dispositions idiosyncrasiques d'une chose individuelle ne serait pas considérée comme très satisfaisante. Lorsque nous nous lançons dans l'étude du monde physique, nous postulons généralement que de telles dispositions sont l'effet de lois qui gouvernent des choses d'une espèce donnée. Et dès lors nous poursuivons notre enquête habituellement jusqu'à ce que nous puissions subsumer ce qui s'est passé sous de telles lois. Mais il est normal chez les historiens, déclare Donagan, de faire des suppositions très différentes quand il s'agit de l'agir humain comme d'ailleurs la plupart d'entre nous le faisons dans la vie quotidienne. On exagère peut-être si on dit que les historiens supposent, en général, que les gens se comportent d'une manière qui leur est particulière. Leurs recherches explicatives, tout de même, inclinent à laisser ouverte la question de savoir s'ils le font ; et leurs conclusions semblent maintenir souvent qu'ils l'ont fait effectivement, pour autant que nous puissions en juger. En ce sens, au moins, soutient Donagan, les historiens s'adonnent à ce qu'on pourrait appeler une présomption de différence individuelle; celle-

9. The Concept of Mind, Hutchinson, 1949, 88-89. 
ci une fois donnée, la valeur des explications par référence au caractère individuel grandit assurément. C'est pourquoi le fameux conseil aux historiens de David Hume prend une résonance si peu historique à beaucoup d'oreilles. Si vous désirez comprendre les Grecs et les Romains, disait Hume, étudiez bien les Français et les Anglais. ${ }^{10}$ Mais, en histoire, on ne peut pas appuyer les explications basées sur le caractère individuel en citant ce que d'autres hommes ont fait dans des circonstances semblables. Ce qu'on doit trouver, si on veut offrir des explications de ce genre, c'est ce que ces mêmes hommes firent, même dans des circonstances toutes différentes.

Dans ses ouvrages plus récents, Donagan s'éloigne d'un pas de plus de l'approche hempelienne, et d'une manière qui soulève de profondes questions métaphysiques d'importance ultime. Les explications qui sont faites par référence au caractère individuel, selon la théorie que je lui ai attribuée jusqu'à ce point, considèrent les hommes d'une façon qui les distingue des objets naturels en ce sens qu'elles prennent au sérieux leur individualité en essayant de les expliquer. Mais les historiens, ajoute maintenant Donagan, sont prêts à traiter les hommes différemment des objets naturels à un autre égard - encore une fois comme la plupart d'entre nous. Ils sont prêts à supposer, non seulement qu'ils se distinguent d'une manière qui donne aux explications par le caractère un intérêt exceptionnel, mais aussi que leurs modes individuels d'agir peuvent changer. En effet, ils supposent que les gens peuvent eux-mêmes changer leurs modes d'agir grâce à leur pouvoir de décider de leurs objectifs et de leur politique d'action. Par conséquent, nous ne devons pas nous attendre à pouvoir expliquer ce que les agents historiques font par référence à leur nature individuelle, si par celle-ci nous entendons un ensemble de dispositions permanentes et naturelles.

En essayant de comprendre ce que les agents historiques ont fait, nous nous appuyons donc non seulement sur une présomption de différence individuelle mais aussi sur la présomption d'un pouvoir de choix. Cela donné, nous ne pouvons attribuer à un agent historique une certaine disposition à agir qu'aux moments où nous avons raison de croire qu'il choisit d'agir de cette manière. Et dès lors la notion d'une disposition se trouve si éloignée du sens

10. Enquiry Concerning Human Understanding, VIII. 
qu'elle revêt pour les objets physiques qu'il serait préférable d'abandonner l'usage de ce terme. Bien sûr, nous croyons souvent avoir des raisons évidentes pour attribuer aux hommes une façon d'agir à long terme, à laquelle nous pouvons nous référer en expliquant ce qu'ils font. L'explication des actes des Danois par référence à leur politique établie - leur politique de piller jusqu'à ce qu'il n'y ait plus de butin - emploie une notion assez près de celle d'une disposition permanente de conduite. On pourrait en dire autant d'une explication des actes d'un politicien qui le décrirait comme un homme jaloux ou vindicatif. Mais il y a des explications d'actions en histoire qui ne sont pas explications par le caractère en ce sens, - celles qui se concentrent sur la décision d'un agent dans un cas particulier. Comment comprendre la structure des explications de ce genre?

La réponse de Donagan à cette question fut d'abord élaborée dans un article de 1964 intitulé " The Popper-Hempel Theory Reconsidered "." Dans cet écrit il discute une explication de la décision de Brutus de se joindre au complot de Cassius : que Brutus aurait pris la résolution de protéger la république romaine à tout prix, et qu'il en serait arrivé à croire que César présentait une menace pour elle que seule sa mort pourrait conjurer. Ce qui explique l'action en ce cas, remarque Donagan, ce sont les intentions et les croyances de l'agent. Celles-ci étant données, ainsi que certaines présuppositions portant sur son habilité et les occasions qui s'offriraient, ce que fit Brutus devait s'ensuivre par une nécessité logique. $\grave{A}$ noter que ce sont les intentions et les croyances de l'agent au moment d'agir qui sont ici pertinentes. Car il n'est pas plus nécessaire qu'il les ait eues à d'autres moments qu'il n'est nécessaire pour lui de les avoir partagées avec des hommes de type similaire.

On ne peut guère douter du fait que ce que Donagan décrit soit un genre d'explication auquel les historiens ont habituellement recours, - surtout au cours de récits historiques. Je crois que Donagan a raison aussi de soutenir que c'est le type d'explication auquel pense Collingwood quand il voit dans la compréhension de l'histoire une "repensée " des pensées des agents du passé, - le but de cette reprise étant de voir dans les actions de

11. History and Theory, IV, 1964, 3-26, et dans W.H. Dray (éditeur), Philosopbical Analysis and History, Harper and Row, 1966, 127-159. Notez aussi " Alternative Historical Explanations and their Verification", The Monist, LIII, 1969, 58.89. 
ceux-ci l'expression de ce qu'ils pensaient. En vérité Donagan présente son analyse comme étant simplement une formulation nouvelle, quoique en termes un peu plus formels, de ce que Collingwood lui-même essayait de dire. L'approche méthodologique de ce genre d'explication, constate Donagan, consiste à élaborer une " hypothèse de pensée " qui entraînerait logiquement l'accomplissement des actions concernées. La méthode, autrement dit, sera "hypothético-déductive ", d'une manière tout à fait analogue aux procédés utilisés dans d'autres types d'enquêtes, même en sciences naturelles.

Cette analyse de Donagan aura à faire face, je crois, à deux objections principales. Premièrement, sa thèse sur la structure interne d'une telle explication sera probablement contestée. On va dire qu'elle demeurera incomplète, logiquement, s'il lui manque une prémisse ou un principe général qui lierait la façon de penser indiquée à la façon d'agir qu'on veut expliquer. Et cela, comme a prétendu Hempel, exigerait une loi empirique. La réponse de Donagan à ce genre d'attaque me paraît assez forte. Il concède que toute explication a besoin d'un principe général ; mais il nie que, dans ce cas, ce serait une loi empirique. Car ce qui donne aux intentions et aux croyances une valeur explicative n'est pas le fait qu'elles sont suivies d'actions d'un type donné. C'est que nous percevons entre penser d'une certaine façon et agir d'une certaine façon un lien logique ou rationnel. Dans le cas de Brutus, par exemple, les pensées admises comme explicatives étaient sa résolution de protéger la république à tout prix, et sa croyance que la protection de celle-ci ne pouvait être assurée que par la mort de César. Ce qui lie ces pensées avec l'action de se joindre au complot de Cassius, selon Donagan, est le principe général : si une personne est résolue d'atteindre un certain but à tout prix, et reconnaît qu'une certaine action est nécessaire à cette fin, elle l'accomplit. Mais ce principe n'est pas une loi empirique ; c'est une affirmation analytique qui est dérivée de ce que l'on entend par de telles résolutions et croyances. Cela se manifeste par le fait que seul le refus de participer au complot de la part de Brutus - le refus de saisir le moyen d'atteindre le but allégué - permettrait de conclure, ou bien qu'il n'était pas vraiment résolu de protéger la république à tout prix, ou bien qu'il ne croyait pas vraiment que la mort de César y fût indispensable. 
Donagan me semble ici être sur la bonne voie - et cela parce qu'il se demande avec plus d'exigence que Hempel ce qu'on voudrait démontrer par une explication en termes de la pensée d'un agent. Néanmoins, son analyse est peut-être un peu exagérée. Il affirme, comme il l'a fait en ce qui concerne les explications dispositionnelles, qu'une bonne explication de ce genre doit être déductive. Par conséquent, ce modèle aussi risque de devenir inapplicable à la plupart des exemples historiques. Même dans l'exemple qui sert de paradigme à Donagan, il est vraiment difficile de formuler l'explication en termes tels que la conclusion «Brutus s'est joint au complot " dérive d'une nécessité déductive. Donagan lui-même concède qu'on ne peut pas la faire dériver des seules affirmations des intentions et des croyances de Brutus. On a besoin, en plus, d'assurances sur ses capacités et sur ses chances : par exemple, il faut savoir qu'il n'est pas ivre ou incarcéré. Et on a besoin de beaucoup d'autres garanties avant de dire que la conclusion s'impose strictement. Il faut nous assurer que Brutus savait qu'il y avait un complot; qu'il croyait que celui-ci aurait une bonne chance de succès ; qu'il doutait que ce complot puisse réussir sans son aide; qu'il n'avait pas de raisons de s'attendre à la mort de César autrement. Il faudrait savoir que Brutus n'était pas la victime au dernier moment d'un accès de faiblesse de volonté ; qu'il n'a pas oublié sa résolution sous la pression d'autres affaires (car, logiquement, il est bien possible d'avoir une résolution et de manquer de l'exécuter dans un tel cas) ; qu'il a reconnu le moment d'agir quand ce moment est arrivé, etc. De plus, il faudrait qu'il soit vrai, comme point de logique conceptuelle, que, si aucune de ces nombreuses (peut-être indéfiniment nombreuses) assurances supplémentaires ne faisait défaut, le simple fait de ne pas agir de la manière appropriée rendrait invalide la prétention que Brutus était résolu à tout prix.

Et même si on pouvait dans un tel cas obtenir autant d'assurances qu'il serait nécessaire, il faut se demander dans combien d'occasions un historien pourrait s'attendre à trouver des agents résolus à agir à tout prix. Si cela arrive rarement, on ne devrait pas s'attendre normalement à donner des "explications par la pensée " déductives. Cependant, le point important pour Donagan serait le suivant : que, si, en réponse à une demande de rendre une explication déductive, on augmentait progressivement les conditions explicatives, ce qu'on ajouterait ne serait pas des lois empiriques; ce 
serait, au contraire, des considérations du genre déjà indiqué. On construirait une explication plus rigoureuse, mais encore donaganienne. Il n'est pas question de la remplacer par une explication d'un type différent - de type hempelien, par exemple. Et si, à la fin, l'augmentation des conditions la laissait toujours non déductive, les conditions citées seraient explicatives quand même à cause du sens des concepts employés. Que Brutus au moins s'inquiétait de l'avenir de la république, et qu'il croyait que César y serait un obstacle considérable, ne justifiaient pas strictement la conclusion qu'il se joindrait à un complot si l'occasion se présentait. Mais s'il le faisait, de telles considérations seraient explicatives - et cela à cause d'un lien conceptuel, sinon rigide, entre penser et agir de la façon indiquée. Jusqu'à ce point, au moins, la thèse essentielle de Donagan semble rester valide.

Le deuxième genre de critique auquel Donagan doit répondre vient de deux de ses hypothèses qui semblent être en opposition l'une à l'autre. La première est que les historiens supposent d'une manière caractéristique, et avec raison, que les hommes peuvent changer leur manière d'agir par leurs décisions ou, si l'on préfère, leur pouvoir de choix individuel. La deuxième est que ces mêmes historiens, quand ils offrent des explications des activités choisies, peuvent toutefois présenter leurs conclusions comme bien fondées empiriquement. La première affirmation laisse ouverte la possibilité que les actions d'un homme, à un moment particulier, puissent être une expression d'intentions et de croyances complètement dissociées de ses actions en d'autres temps. Mais la deuxième semble demander que les agents, dans la mesure où leurs actions sont explicables, n'agissent pas selon des intentions et des croyances qui ne s'expriment que dans une action singulière. Car, en supposant qu'ils le fassent, il n'y aura manifestement, en dehors de l'action elle-même, aucune voie d'accès à ce que sont en réalité ces intentions et ces croyances. Et s'en remettre à cette unique source ne serait pas atteindre une conclusion d'une manière empiriquement bien fondée. Autrement dit, si la procédure de l'historien, quand il établit une explication par la pensée, est hypothéticodéductive, comme Donagan l'a dit, elle impliquera la mise à l'épreuve d'une "hypothèse de pensée " qui consisterait en une vérification des affirmations qu'on pourrait en tirer concernant d'autres choses que l'agent aurait dites et aurait faites; mais la légitimité d'une telle procédure est exactement ce que le point de vue méta- 
physique de Donagan sur l'action remet en question.

Donagan lui-même comprend bien le problème; et il y répond de la façon suivante. La supposition que les intentions et les croyances des gens peuvent changer, indique-t-il, n'est pas du tout la supposition qu'elles sont souvent, ou parfois, ou même jamais momentanées. On a besoin de preuves concrètes pour justifier la prétention qu'il y eut un changement des intentions ou des croyances d'un agent ; ce n'est jamais une affaire de pure supposition. Cela me semble assez acceptable comme hypothèse de travail. Mais il laisse l'analyse de l'explication historique dans une position assez embarrassante. Ce qui est évident c'est que, si un historien essaie d'interpréter une certaine action dans le passé, il doit toujours être ouvert à l'hypothèse que les intentions de l'agent aient pu changer. Quelquefois il pourra peut-être découvrir quand cela est arrivé ; quelquefois non. Quelquefois il pourra peut-être expliquer un changement par référence à des intentions plus larges ; quelquefois non. Ce que reconnaît la théorie de Donagan à la fin, même si cette fois je suis enclin à l'accuser d'un excès de modération, - c'est donc une incertitude endémique des explications de l'action en histoire. À noter que c'est une caractéristique qui n'est pas affectée du tout par le succès ou le non-succès des tentatives de les structurer dans une forme déductive.

\section{III}

L'insistance de Donagan sur le caractère individuel d'une grande partie de l'explication historique met le modèle de Hempel très directement au défi. Elle conteste sa thèse centrale concernant la structure logique interne qui seule permet de considérer comme formellement complète une explication, déductive ou inductive.

La seconde position philosophique que je me propose de mettre en rapport avec celle de Hempel la conteste d'une façon moins directe, au moins au début. Hempel ne s'est pas sérieusement demandé si une explication qui satisfait aux exigences de l'explication par une loi de couverture (" covering law") établit en même temps ce qu'on peut appeler correctement la cause, ou les causes, de ce qui s'est passé. Il s'est exprimé, en effet, comme si la question de savoir si toutes les conditions explicatives étaient des 
causes n'était qu'une question de terminologie qui n'avait pas d'intérêt particulier. Donagan, lui non plus, ne s'est pas directement demandé si une explication qui a invoqué les croyances et les intentions d'un agent, ou même un aspect de son caractère, était une explication causale. Bien sûr, il nierait qu'elle soit causale dans un sens propre à la science naturelle - un sens " humien ". Mais, comme l'avait fait Collingwood avant lui, il est porté à dire que les croyances et les intentions d'un agent constituent conjointement, dans un sens spécialement historique, la cause d'une action qui les exprime. Aucun des deux théoriciens n'explique ce qui est impliqué dans les explications qui isolent, de toutes les conditions acceptées comme pertinentes à une action ou à un événement, une condition, ou plusieurs, qu'on peut appeler sa cause. Cependant c'est précisément cette question, la question visant à identifier la condition distinctement causale, qui a engendré quelques-unes des polémiques les plus animées entre les historiens.

Les philosophes qui, à mon avis, ont dit les choses les plus éclairantes à propos de l'aspect spécifiquement causal de l'explication, n'ont pas du tout eu, comme préoccupation dominante, de faire de la philosophie de l'histoire. Ce sont deux philosophes oxfordiens du droit, H.L.A. Hart et A.M. Honoré, auteurs conjoints d'un livre, Causation in the Law $w^{12}$, qui explore de façon détaillée et impressionnante comment les avocats font la distinction entre les causes et les autres conditions nécessaires. Hart et Honoré signalent, bien que seulement en passant, que les historiens et les avocats appliquent presque les mêmes principes dans leurs analyses causales. Cela me paraît juste en grande partie.

Au début de leur étude, Hart et Honoré se servent d'un exemple, assez banal mais éclairant, bien qu'il concerne un événement naturel ${ }^{13}$. Imaginez une explosion dans une fabrique de produits chimiques. Lorsqu'un tel événement a lieu, il y a beaucoup de conditions qu'on sait être nécessaires, et qui appartiennent, dans un sens large, à son explication. La présence de l'oxygène constitue une telle condition. Néanmoins il serait étrange de prétendre que cette présence fut la cause de l'explosion, surtout si l'on sait que, parmi les autres conditions, il y a eu un mégot échappé par un fumeur négligent. Celui-ci est évidemment un candidat

12. Clarendon Press, 1959.

13. Op. cit., 10. 
préférable au statut de cause. Pourquoi? Hart et Honoré soutiennent - et je suis d'accord avec eux - que nous ne pouvons pas prétendre avoir compris le concept de cause, et donc ce qui est impliqué dans une explication causale, tant que nous ne pouvons pas répondre à cette question. Il nous faut savoir si la distinction entre cause et pures conditions relève de principes consistants. Nos auteurs constatent que de tels principes sont vraiment appliqués, même si leur nature se trouve souvent obscurcie par leur contexte.

On pourrait formuler très simplement ces principes en disant que, pour être causess, des conditions doivent êire, soit quelque chose d'anormal, - un événement anormal ou une liaison anormale d'événements, - soit des interventions provenant d'agents humains ou de groupes, qui peuvent être classifiés comme volontaires. La raison pour laquelle l'action de fumer fait figure de candidat si fort dans le cas de l'explosion, c'est que nous pouvons supposer qu'il est anormal de fumer dans une fabrique de produits chimiques. Nous pouvons supposer le contraire à propos de la présence de l'oxygène, qui est donc reléguée au statut d'une condition auxiliaire - une partie des circonstances à l'intérieur desquelles la cause a agi. $\mathrm{Si}$ on change ces suppositions, les jugements causaux changent aussi. Imaginez un procédé de production qui exigerait l'exclusion de tout oxygène. Sa présence serait, en ce cas, anormale, et s'il s'en infiltrait quelque peu, et s'il y avait une explosion, l'oxygène serait un candidat excellent au titre de cause. Ce serait le cas, on doit le remarquer, même si les deux explosions étaient chimiquement identiques. Et si l'on découvrait que l'oxygène a été infiltré délibérément par un incendiaire, de telle sorte qu'une intervention volontaire fût impliquée aussi bien qu'une anormalité, son statut causal serait encore plus fermement établi. Pour des raisons semblables, si quelqu'un, marchant devant un bon tireur qui est en train de tirer, se trouve fatalement blessé, on peut dire qu'il a causé sa propre mort. Mais si le tireur, dans une situation physiquement identique, vise l'homme intentionnellement, c'est au tireur, et non pas à sa victime, que l'on attribue le rôle de cause."

Les explications causales en histoire procèdent-elles avec des principes semblables? Hart et Honoré ne donnent pas d'exemples

14. Op. cit., 40 . 
tirés d'ouvrages d'histoire; mais il n'est pas difficile de trouver des historiens qui, pour appuyer leurs revendications causales, se servent d'une argumentation analogue. On en trouve un bon exemple dans la discussion entre deux historiens anglais bien connus, A.J.P. Taylor et Hugh Trevor-Roper, à propos des causes de l'éclatement de la guerre en 1939. Taylor, dans son livre intitule The Origins of The Second World War, ${ }^{15}$ qui lui a valu beaucoup de notoriété, s'en prend à ce qu'il considère la façon de voir la plus acceptée depuis la fin de la guerre elle-même : celle de chercher les causes du conflit, dans une mesure beaucoup plus large qu'on a l'habitude de le faire, dans les croyances, les ambitions et les actions d'un seul individu, Adolph Hitler. Taylor constate, au contraire, qu'on a exagéré le rôle d'Hitler dans le processus entier. Il soutient que les vraies causes de la guerre résident dans une série de bévues qui ont été commises autant, ou peut-être plus, par les adversaires d'Hitler, et en particulier par les politiciens britanniques et français qui n'acceptaient pas de renoncer à l'impossible traité de Versailles. Trevor-Roper soutient la thèse plus traditionnelle ${ }^{16}$ et accuse Taylor de fausse représentation en essayant de la réviser. Ce qui nous intéresse, c'est l'espèce de fausse représentation à laquelle Trevor-Roper semble penser. Il n'y a pas beaucoup de différence entre les deux historiens en ce qui concerne - peut-on dire - les "faits" fondamentaux en question. Ce qui les divise, et ce qui explique en dernière analyse les conclusions causales différentes qu'ils en tirent, ce sont leurs conceptions différentes de ce qui est normal et de ce qui est anormal dans les circonstances, et leurs jugements également différents sur ce qui était volontaire et ce qui ne l'était pas.

En soutenant sa propre position, Trevor-Roper considère donc qu'il est nécessaire d'attaquer directement la représentation que Taylor se fait d'Hitler comme un homme d'état allemand ordinaire, dans la tradition de Streseman et de Bruning, dont les politiques ne seraient pas substantiellement différentes de celles de ses prédécesseurs. Il n'accuse jamais Taylor d'avoir prétendu - ce qui aurait été ridicule - qu'Hitler lui-même n'a pas joué un rôle essentiel dans les événements qui ont abouti à la guerre. Ce qui gêne Trevor-Roper, c'est que Taylor soutient, comme raison de

15. Hamish Hamilton, 1961.

16. "A.J.P. Taylor, Hitler and the War ", Encounter, XVII, 1961, 88-96. 
nier qu'Hitler fut la cause du conflit, que le but du leader allemand ne fut rien d'autre que la restauration de la position naturelle de son pays dans le système européen - une position, dit Taylor, qui avait été changée artificiellement par le traité de Versailles. D'après Taylor, ce qui fut étrange, et ce que l'on a beaucoup plus raison de prendre pour cause éventuelle du conflit, c'est le manque d'un réalisme semblable de la part des Britanniques et des Français - un jugement que Trevor-Roper refuse totalement. Ce qui importe à notre recherche n'est pas de savoir lequel des deux historiens propose le jugement le plus acceptable. Ce qui est important, c'est plutôt le concept de cause qui se trouve à l'oeuvre dans leur désaccord. Car même s'ils ne sont pas d'accord sur le causes de la guerre, ils sont d'accord sur les critères à utiliser pour juger la cause d'un fait historique. Tous les deux estiment que, pour être cause, il faut qu'une condition soit, à la fois, nécessaire par rapport à ce qui s'est passé et anormale par rapport aux circonstances. En effet, s'ils n'étaient pas d'accord sur ces critères, toute leur discussion - qui à première vue semble assurément être un désaccord réel sur une question historique - ne serait qu'un échange de propos équivoques.

L'idée d'une cause consistant en une intervention volontaire ne se présente pas moins clairement dans la dispute entre les deux historiens. Comme le remarque Trevor-Roper, avec une irritation croissante, Taylor caractérise presque toujours Hitler comme un homme qui répond aux événements au lieu de les provoquer. La crise de 1938 en Autriche, nous assure-t-il, a été déclenchée par Schuschnigg, non par Hitler. La crise des Sudètes, qui s'est produite plus tard dans la même année, a été le fait des Nazis sudètes; Hitler n'a fait que l'exploiter une fois qu'elle fut provoquée. Sa répudiation subséquente de la répartition de la Tchécoslovaquie lui fut imposée par les manoeuvres maladroites du Président Hocha. La crise polonaise lui fut également imposée par Beck. Encore une fois nous ne devons pas trop nous préoccuper de la justesse de ces jugements historiques eux-mêmes. Ce qu'il faut remarquer, c'est que les deux historiens - Taylor qui les émet et Trevor-Roper qui les rejette - concèdent qu'ils sont pertinents à la question de savoir si Hitler lui-même a été cause de la guerre. Si Taylor pouvait vraiment démontrer que les contributions d'Hitler au processus qui a éventuellement mené aux hostilités n'étaient pas libres, mais forcées, Trevor-Roper concéderait qu'il faut chercher ailleurs, 
dans d'autres actions et d'autres événements, les causes de la guerre. Mais selon Trevor-Roper, c'est une chose que Taylor ne peut pas faire.

Si cet exemple illustre quelque peu ce que font les historiens, - et je crois qu'ils le font effectivement pour une assez grande partie de leur recherche, - il nous force à reconnaitre explicitement un aspect logique de l'explication causale en histoire qui pourrait être un peu surprenant. C'est la relativité de celle-ci par rapport au point de vue apporté par l'historien dans son enquête. On peut comprendre cela plus clairement peut-être dans le cas où le choix de la cause suit le principe d'anormalité. Car qualifier quelque chose d'anormal, c'est employer implicitement un standard de comparaison; et par rapport à deux standards différents, évidemment, il faudrait juger le même événement respectivement normal et anormal. Dans le cas de l'explosion, par exemple, si nous nous demandons si la présence de l'oxygène fut normale ou anormale, nous ne pouvons pas résoudre ce problème en cherchant seulement du côté des données statistiques. Il nous faudrait d'abord décider si nous nous intéressons à la présence de cette substance dans les fabriques en général, dans les fabriques d'un genre particulier, dans les fabriques d'un endroit spécial, dans les fabriques d'une période déterminée, etc. Ce n'est pas la statistique qui permet de savoir quel standard employer. Il s'agit d'une question qui concerne les intérêts du chercheur - ou, si vous voule $z$, son système de valeurs. Il y a un problème semblable dans la dispute entre Taylor et Trevor-Roper à propos des causes de la seconde guerre mondiale. Pour le dire dans le langage de TrevorRoper, la conclusion causale de Taylor envisage comme position "naturelle" ou "réelle" les pouvoirs relatifs des nations européennes au printemps de 1918, la dernière année de la première guerre, quand l'Allemagne était au sommet de son succès à l'est et à l'ouest. C'est par comparaison avec cette situation que Taylor voit dans le système de Versailles une anomalie - une situation artificielle ou anormale. Trevor-Roper ridiculise bien la remarque faite par Taylor à propos du système de Versailles, selon laquelle celuici manquait de validité morale, mais il ne met pas en lumière le fait que l'historien, dès qu'il choisit un standard de comparaison dans un tel cas, s'adonne nécessairement à des considérations morales. La question qui se pose est la suivante : quelles étaient les attentes raisonnables, et peut-être même les droits, d'une puissance 
telle que l'Allemagne, compte tenu de son histoire récente et de ses possibilités, dans le système européen de la première moitié du vingtième siècle? Cette question n'est pas arbitraire: c'est la nature du concept de cause qui la rend pertinente dans la recherche en question.

Le choix d'une action comme cause, au nom de son caractère volontaire, peut également être relatif à un point de vue et impliquer un jugement moral. Comme pour le cas de l'anormalité, on pourrait croire qu'il y avait un moyen objectif de rendre le jugement pertinent - par exemple, en demandant laquelle, parmi les actions susceptibles d'être des conditions nécessaires de ce qui s'est passé, était la moins déterminée par ses conditions antécédentes. Mais en faisant cela, on comprendrait mal le sens dans lequel l'historien a besoin de savoir si les actions qu'il veut considérer sont forcées. On supposerait que la question à traiter en est une de dé. terminisme versus indéterminisme. Mais ce n'est pas le cas. Il s'agit d'une question sur la nature morale des choix ouverts aux divers agents, quand ils envisagent les circonstances dans lesquelles ils se trouvent. La question de savoir si Hitler fut forcé ou non d'envahir la Pologne ne concerne pas ses tendances psychologiques dans les circonstances. Encore moins a-t-elle rapport au problème de l'inévitabilité historique. Il s'agit de la légitimité des intérêts qui, selon Hitler, se trouvaient menacés par les Polonais. Ce n'est que dans le cas où ces intérêts auraient été légitimes qu'on pourrait dire qu'Hitler fut forcé d'agir. Autrement dit, le concept d'une action non volontaire, et donc aussi d'une action volontaire, dans le sens qui est pertinent, est lui-même un concept quasi moral. Et parce que la spontanéité, dans ce sens, est pertinente à la distinction entre causes et conditions auxiliaires, le concept de cause de l'historien est aussi un concept quasi moral.

On peut trouver un peu ironique que le problème de la relativité des conclusions en histoire soit soulevé à propos de ses explications causales. Car les relativistes historiques, et parmi eux surtout les philosophes, ont généralement trouvé ailleurs la relativité de l'histoire : par exemple, dans la décision de l'historien d'inclure ou d'exclure certains détails en écrivant un récit particulier, - le soidisant problème de "sélection ", - ou dans son choix du langage pour caractériser les actions ou les événements du passé. La découverte des causes, surtout si on la comprend à la Hempel, comme la 
découverte que certaines lois étaient implicites dans un cas examiné, a généralement été prise comme type de recherche qui, logiquement, n'accorde aucune place aux valeurs du chercheur. Il vaut donc la peine peut-être d'ajouter que, même si Hempel a raison et si Donagan a tort en ce qui concerne la fonction des lois dans l'explication historique, une telle explication sera encore relative à un point de vue, pourvu qu'on pousse le raisonnement jusqu'à l'identification des causes. Car même si une cause est, disons, une condition nécessaire, qui complète un ensemble de conditions suffisantes (l'analyse la plus acceptable, je crois, de celles qu'on peut attendre d'une approche purement hempelienne), ce n'est pas là tout ce qu'elle est - du moins, pas dans le vocabulaire des historiens. Comme la plupart d'entre nous, ceux-ci conçoivent les causes, le plus souvent, comme conditions qui font que les choses arrivent ; et ils ont des critères pour identifier de telles conditions -- des critères qui reflètent, sans doute, des notions métaphysiques, assez larges et assez obscures, d'activité et de passivité, d'agent et de patient, mais qui dans leur application aux processus sociaux reçoivent des interprétations qui posent des questions d'intérêt humain fondamental. Des analyses aseptiques, en termes seulement de conditions nécessaires et suffisantes, ne commencent même pas à élucider ces critères.

On doit donc regarder la théorie de Hempel comme, au moins, sérieusement incomplète, en tant qu'élucidation du concept d'explication et de compréhension en histoire. Elle ignore toute une dimension des enquêtes causales. Mais ce n'est pas tout. Je ne concéderais pas moi-même, par exemple, que ce que les historiens appellent "la cause " soit conçu, dans tous les cas, même comme une condition qui, parmi autres choses, complète des ensembles de conditions suffisantes. Suivant encore une fois Hart et Honoré, mais allant aussi un peu plus loin, je constaterais qu'on peut attribuer le statut causal, avec une intelligibilité et une convenance indiscutables, aux conditions dont on ne sait pas si elles complètent ou non un tel ensemble, et même à celles dont on croit qu'elles ne le font pas. Pourvu qu'elle satisfasse aux autres critères du choix des causes semblables à ceux que j'ai déjà mentionnés, il suffit à une condition, en certains cas, d'être nécessaire parmi d'autres conditions nécessaires.

Car une cause est quelquefois conçue comme ce qui permet, 
plutôt que ce qui fait, un événement. Dans la loi, comme l'indiquent Hart et Honoré, ce qu'on appelle la cause d'un tort est souvent l'absence de précautions raisonnables contre un tel résultat. ${ }^{17}$ Un geste négligent qui donne à un voleur l'occasion de s'introduire dans une maison, par exemple, pourrait être jugé la cause de la perte qui en résulte, même si on ne croit pas que son intrusion a nécessairement, ou même probablement, suivi ce geste, étant données n'importe quelles autres conditions, connues ou inconnues. Dans un tel cas, ce qu'on dit dépend de la nature du risque et de l'importance du résultat. Quand l'importance est grande, l'action négligente peut atteindre le statut de cause, même s'il était peu probable que dans les circonstances elle soit exploitée. De même un commandant militaire qui donne à son adversaire une occasion de l'attaquer, même si cette sorte d'occasion ne risque pas d'être exploitée plus qu'une fois sur douze, pourrait être accusé, non seulement par son gouvernement mais aussi par son historien, d'avoir de cette manière causé sa propre défaite.

Ce qui est encore pire pour la théorie de Hempel, c'est que ce qu'on regarde comme effet pourrait être jugé en même temps, et sans tomber dans l'incohérence, comme événement improbable; et je ne veux pas dire improbable dans quelque sens général, mais dans un sens qui s'applique au cas particulier, la cause étant donnée. Cela exclut même la seconde théorie de Hempel, la moins exigeante, qui impliquerait assurément que la compréhension s'évanouit au point où la probabilité d'un événement atteint le point zéro. En effet, dans le sens que nous examinons maintenant, - et il y a des gens qui le trouveront scandaleux, - le concept de cause se présente non seulement comme un concept relativiste, mais aussi comme un concept indéterministe. Il s'agit de possibilités, non de probabilités, encore moins de nécessités. Peut-être y a-t-il beaucoup de gens qui concéderaient que, dans un certain sens d'explication, un événement pourrait être expliqué en montrant ce qui l'a rendu possible. J'ai moi-même appelé ailleurs "explication par les conditions de possibilité " (" explaining how possibly") une variété de ce type d'explication, variété qui exige la réfutation d'une supposition d'impossibilité, ce qu'on fait en indiquant la présence d'une condition nécessaire qui a été, dans le contexte, mise en question. Mais ma position actuelle va plus loin : c'est qu'en un sens

17. Causation in the Law, 55.57. 
parfaitement familier, et dans un contexte d'intérêt approprié, ce qui rend une action ou un événement possible peut être considéré sa cause. Bien sûr, un jugement causal dans ce sens n'entraine pas en soi l'indéterminisme ; on peut rester satisfait, à un certain point d'une recherche, simplement de ne pas poser une question qui exigerait une réponse détermiriste. Mais ce qui est intéressant, c'est qu'un indéterministe - et il y en a beaucoup en ce qui concerne les actions humaines - peut se livrer à ces jugements causaux sans tomber $\mathrm{du}$ tout dans l'incohérence. Les indéterministes ne manquent pas entièrement de ressources explicatives, contrairement à ce que l'on pourrait croire en prêtant trop d'attention à la sagesse courante.

Avant d'abandonner cette dernière question, je devrais peutêtre faire remarquer que même certaines causes, qu'on représente comme nécessitantes, pourraient être conçues comme indéterministes dans leur opération, si des philosophes comme Donagan ont raison. Comme je l'ai signalé plus tôt, Donagan et Collingwood soutiennent tous deux que ce qu'on peut proprement appeler la cause d'une action en histoire consiste dans les pensées qu'y exprime un agent. Une autre façon de formuler leur position à mon sens est la suivante: les causes des actions en histoire ne produisent leurs effets - et ne sont ainsi représentées dans l'explication - que dans la mesure où les agents historiques les conçoivent d'une certaine façon, leurs intentions étant données. Si les agents, comme le soutient Donagan, ont un pouvoir individuel de choix, - si leurs intentions ne sont pas complètement déterminées par les conditions extérieures, - leurs actions ne seront pas non plus déterminées par leurs causes. Et il en est de même quand nous parlons, dans le sens courant, d'un agent qui a été forcé d'agir comme Taylor parlait d'Hitler à propos de sa réponse au problème polonais. Néanmoins il y a une différence importante entre ce genre de cause et celui dont je parlais il y a un instant, en ce qui concerne le déterminisme. Dans le cas donaganien, l'explication n'est pas indéterministe sauf en ce sens qu'un de ses éléments l'intention - est considéré comme étant lui-même indéterminé. Ce n'est pas la relation entre ce qui explique et ce qui est expliqué que l'on traite comme indéterministe. Dans le cas des causes qui ne font que rendre possible un événement, c'est tout à fait différent. Ce que l'on tient pour indéterministe, c'est la structure de l'explication elle-même. 


\section{IV}

La troisième position avec laquelle je voudrais comparer celle de Hempel a été discutée notamment par les philosophes américains Arthur Danto et Louis Mink. Tout comme dans le cas de la théorie de l'explication causale de Hart et d'Honoré, il s'agit d'une position qui ne s'oppose pas directement aux thèses de Hempel. Elle met plutôt en relief la pertinence assez limitée de la forme hempelienne d'explication à l'égard de ce que l'on peut appeler une compréhension typiquement historique du passé. Cependant, elle attire aussi notre attention sur d'autres manières caractéristiques, trompeuses sinon fausses, d'appliquer la structure hempelienne à des récits explicatifs en histoire.

On peut aborder l'affirmation centrale de la thèse de DantoMink par la remarque suivante : la théorie hempelienne prend pour acquis que la compréhension des événements du passé chez les historiens les lie aux événements qui les ont suivis. Comprendre un événement historique, entend-on dire souvent, c'est le situer dans une perspective historique - ce qui exige de l'historien, entre autres choses, qu'il observe ses conséquences. Comprendre la Révolution française comme événement historique, c'est apprécier le rôle qu'elle a joué dans l'histoire de la France. Saisir la signification de la Réforme, c'est reconnaître son importance pour l'accroissement du nationalisme, ou pour la renaissance de l'Église catholique. Il est vrai que, dans de tels cas, c'est encore, dans un sens large, des relations causales que l'historien discernera. Mais la direction dans laquelle il les cherchera sera renversée.

L'habitude des historiens de chercher à comprendre les événements à partir de leur avenir, aussi bien que de leur passé, relève sous un aspect distinctif, mais rarement remarqué, de la description historique ordinaire. C'est le reportage des actions et des événements du passé employant des expressions qui renvoient implicitement à ce qui n'a pas encore eu lieu. Un récit historique, par exemple, peut décrire les coups de feu tirés à Fort Sumter comme "la première volée de la guerre civile américaine ". Ou l'historien peut nous dire, à un certain moment, qu'en 1756 est né le compositeur de "La Flûte enchantée ". Mink parle à cet égard des 
"descriptions chevauchantes" ("overlapping descriptions") des historiens, et Danto de leurs "phrases distinctement narratives" ${ }^{18}$ C'est seulement parce que les historiens ne rendent compte d'un événement que longtemps après coup, qu'il leur est légitime de se servir d'un tel langage que l'on pourrait qualifier de "récit implicatif ". C'est cela qui leur permet de voir dans les événements du passé ce qui échappait nécessairement à leurs témoins, et dans les actions ce que leurs auteurs ne pouvaient ni penser ni vouloir.

Un autre aspect des récits historiques qui aide à exprimer l'essentielle rétrospectivité de la compréhension qu'ils cherchent à transmettre, c'est le langage très fataliste qu'ils emploient souvent. Il y a une tendance de la part des critiques philosophiques à ridiculiser l'emploi par des historiens d'expressions telles que "moments décisifs" ou " pas fatidiques". On les considère simplement comme les manifestations d'une tendance chez les historiens assez sympathique, mais tout de même un peu bête - à se laisser gagner par les possibilités dramatiques d'une bonne histoire. En fait, la compréhension distinctement historique est vraiment fataliste, au sens philosophique très précis du mot. Le fatalisme est une version de la doctrine du déterminisme qui représente l'avenir, non pas comme quelque chose que le présent entraînera nécessairement, mais comme quelque chose qui, en un certain sens, existe déjà fixé et immuable. L'historien peut donc en tenir compte d'une manière tout à fait différente d'un avenir qu'on ne peut qu'envisager ou prédire.

L'essentielle rétrospectivité de la connaissance historique comporte, comme implication importante, qu'une telle compréhension de notre propre époque nous est inaccessible. La soi-disant " histoire contemporaine" est impossible : elle exige une connaissance détaillée de l'avenir que peu d'historiens peuvent revendiquer. Une autre implication réside dans le fait qu'aucun récit historique ne peut jamais prétendre être définitif - et cela pour une raison qui s'ajoute à celles qu'on admet communément pour considérer toute recherche empirique comme corrigible. On doit toujours récrire l'histoire, non seulement parce qu'il y aura périodiquement des événements nouveaux, mais aussi parce que la signification des événements passés change réellement. Comme l'a

18. L.O. Mink, "History and Fiction as Modes of Comprehension ", New Literary History, I, 1970, 55-56. A. Danto, Analytical Pbilosopby of History, ch. VIII. 
dit Danto, le passé historique subit, avec le passage du temps, un "alignement rétroactif ". Il s'agit ici d'une forme de relativité qui ne doit pas être confondue avec celle qui fut introduite par la distinction entre cause et pures conditions - une forme à laquelle songent habituellement ceux qui se plaignent de ce que toute histoire est relative au présent. Cette nouvelle relativité accorde-t-elle aussi place aux jugements de valeurs des historiens dans leurs récits? Cela dépend si, en cherchant les conséquences des faits qu'ils exposent, ils appliquent des principes de sélection analogues à ceux que nous avons discernés dans la détermination des causes. Je crois qu'il en est ainsi, bien qu'il ne me soit pas possible, en ce moment, de développer davantage cette question.

Le fait que l'histoire soit rétrospective comporte également des implications pour l'acceptation d'une certaine théorie de la compréhension historique - une théorie, cette fois, qui a été assez populaire parmi les adversaires de Hempel. C'est la doctrine que dans la philosophie anglophone on associe surtout au nom de R.G. Collingwood, pour qui une approche typiquement historique du passé exige de l'historien qu'il s'engage dans une "réactualisation du passé ", ou une "repensée des pensées " des agents dont il étudie les actions. C'est ainsi que Donagan, comme je l'ai souligné, présente comme collingwoodienne son interprétation de la façon dont les historiens expliquent l'action de Brutus de se joindre au complot de Cassius. En attribuant à l'agent des intentions et des croyances explicatives, l'historien lui attribue en même temps un raisonnement pratique qui, si l'on me permet de dépasser un peu les formules exactes de Donagan, rationalise ce qu'il a fait. Cette rationalisation ne doit cependant se faire qu'à partir du point de vue de l'agent. Car un argument qu'on impute à un agent peut uniquement tenir compte de sa situation telle que lui-même l'a conçue, et non pas telle qu'elle a vraiment été (s'il y a une différence), encore moins telle qu'elle pourrait apparaitre à la postérité. En effet, si les cas de ce genre étaient pris comme paradigmes de la compréhension historique (comme ce semble être le cas chez Collingwood, et peut-être aussi chez Donagan), cette compréhension serait exactement le contraire d'un regard rétrospectif sur le passé. Elle consisterait plutôt dans un regard prospectif, à la manière de celui que les acteurs de l'histoire ont nécessairement adópté en agissant. Mais les récits des historiens n'acceptent pas une telle limitation de point de vue. Les théories de type collingwoodien, ou 
"empathique ", ne peuvent donc pas fournir une analyse générale de la notion de compréhension historique qui, en fin de compte, serait acceptable.

Jusqu'ici, je n'ai mentionné explicitement que la découverte des conséquences comme manière d'incorporer dans des récits historiques leur savoir après coup - la rétrospectivité. En réalité, l'historien met au service de la même fonction la reconnaissance de bien d'autres sortes de relations. Quelques-unes de celles-ci ressortent des exemples que j'ai déjà employés. Les coups de feu tirés à Fort Sumter, par exemple, n'ont pas été pris par l'historien pour la cause de la guerre civile américaine, mais pour son commencement. Et quant à la naissance de Mozart, bien qu'elle ait été une condition nécessaire à la composition de "La Flûte enchantée ", on ne prend certainement pas celle-ci pour sa conséquence. Chez les historiens, ce qui arrive plus tôt est vu souvent comme une anticipation, plutôt qu'une cause, de ce qui suit. Des actions d'individus et de groupes sont interprétées comme des révélations ou des signes de conditions beaucoup plus générales. Des événements particuliers sont dépeints comme points culminants ou points décisifs dans les courants soumis à l'étude. Vraiment, les relations qui expriment la rétrospectivité dans les récits des historiens sont, logiquement, extrêmement hétérogènes.

Il manque souvent aux Hempeliens, il me semble, de remarquer tout cela ; ou, s'ils le remarquent, ils ne le tiennent pas pour très significatif. On en trouve un bon exemple chez Morton White. Dans son livre Foundations of Historical Knowlegde, White se demande quelle est la différence entre une chronique pure, qui ne fait rien d'autre que rapporter certains événements selon leur ordre chronologique, et un vrai récit historique, qui vise à rendre compréhensible un tel reportage ${ }^{19}$. Cette différence, il la voit dans le fait que le récit ajoute aux événements rapportés par la chronique des relations causales. L'idéal d'intelligibilité d'un récit, pour White, réside donc dans la chaîne causale, selon laquelle chaque événement est l'effet de son prédécesseur et la cause de son successeur. Mais un tel idéal requiert de l'historien en même temps trop et trop peu. Sans doute il y a des chaînes causales en histoire : les événements menant à l'éclatement de la première guerre mondiale (si vous me pardonnez un autre exemple de ce genre)

19. Harper and Row, 1965, ch. VI. 
viennent assez près de fournir au modèle de White une certaine application. Mais on trouve rarement dans les travaux des historiens de telles chaînes contenant plus qu'une demi-douzaine de maillons ; et la plupart des récits, de toute façon, dépistent des séquences liées différemment, et de façon plus subtile.

Ce qui est étrange, c'est que Danto, qui est beaucoup moins hempelien que White, parle aussi parfois comme si la causalité fournissait, dans un récit, le seul genre de lien, bien que sa conception des maillons causaux eux-mêmes se rapproche quelque peu de ce que les historiens peuvent s'attendre à trouver. L'idéal pour Danto consiste dans un récit où les changements dans un sujet central sont tous expliqués causalement, bien que les causes ellesmêmes puissent ne pas recevoir le même type d'explication. D'après White, par exemple, un récit idéal de l'approche de la guerre en 1914 chercherait une série d'occurences comme la suivante: l'assassinat de l'archiduc causant l'envoi de l'ultimatum autrichien, lequel aurait causé à son tour la mobilisation des Russes, et celle-ci l'intervention des Allemands, etc. Pour Danto, une série causale peut prendre une structure un peu différente. Dans les premières étapes de la guerre elle-même, par exemple, il lui suffirait de trouver une succession de la forme suivante : l'invasion de la Belgique causée par la difficulté d'entrer autrement en France ; l'insuccès des Allemands dans leur marche sur Paris causé par la fermeté des Alliés sur la Marne; l'installation des deux forces dans la guerre des tranchées causée par le manque d'imagination des commandants des deux côtés, etc. Mais même ce deuxième modèle, que j'ai ailleurs appelé modèle de "causal input ", du fait que les causes sont introduites dans une série comme de l'extérieur, - est manifestement trop restrictif pour convenir à la plupart des récits explicatifs en histoire. Et on ne l'améliorerait que marginalement, me semble-t-il, si on remplaçait les relations strictement causales - comme le fait, par exemple, W. B. Gallie dans son livre Philosophy and the Historical Understanding ${ }^{20}$ - par des relations d'un type plus faible: de simples relations de conditions nécessaires, peut-être, bien que Gallie ait probablement raison de prétendre que, souvent, de telles relations sont assez fortes pour soutenir la continuité et l'intelligibilité d'un récit historique.

20. Schocken, 1964 
Nous manquerions l'essentiel de la connaissance historique si nous ne nous libérions pas de cette idée qu'elle consiste uniquement à retracer des relations même quasi causales. Nous manquerions également quelque chose d'essentiel si nous supposions qu'elle se réduit simplement à retracer morceau par morceau des relations de n’importe quelle sorte entre des événements spécifiques. Elle implique quelque chose d'autre, dont un indice nous est fourni par l'emploi dans le langage des historiens d'expressions comme " commencement ", "fin ", " apogée ", " point culminant ". Comme Danto l'a dit, l'un des buts principaux de la recherche historique est de découvrir comment les actions et les événements individuels entrent dans la constitution de "totalités temporelles" plus vastes. Comme Mink préfère le dire : la connaissance historique vise une "unité synoptique" dans un sujet étudié. Ainsi un ensemble de phénomènes culturels très diversifiés, répandus sur une surface géographique considérable, sont envisagés dans leurs relations nombreuses comme étant "La Renaissance ". Un historien comme Johan Huizinga dessine ce qu'il nomme "Le déclin du moyen âge ». D. G. Creighton raconte la montée et la chute de "L'empire commercial du St-Laurent ». Quelques-uns des exploits les plus impressionnants de la recherche historique ont consisté en l'élaboration de visions synoptiques de ce genre. Les questions qui font découvrir aux historiens l'intelligibilité qu'ils prétendent découvrir dans le passé sont souvent moins formulées en termes de "Pourquoi cela a-t-il eu lieu?", qu'en termes de "Qu'est-ce qui s'est vraiment passé?"

Le fondement logique de la connaissance synoptique réside dans la relation entre la partie et la totalité, non pas entre l'antécédent et le conséquent. Bien sûr, puisque la plupart des totalités les plus intéressantes en histoire se composent de parties non simultanées, on aura besoin, pour les faire apparaître, de retracer des relations temporelles de différentes espèces. Mais la connaissance que l'on cherche à atteindre par des récits synoptiques dépasse de beaucoup la somme des explications détaillées qu'ils contiennent. Elle exige une "vision d'ensemble " dans laquelle s'il m'est permis de citer Mink encore - l'idée d'une succession temporelle telle quelle disparaît, ou ne reste qu'à titre de résidu. Sachant la fin du commencement, la connaissance historique échappe à la " rivière du temps" et gagne une sorte de vue "aérienne ". D'où l'importance des " descriptions chevauchantes " qui 
sont si caractéristiques des récits historiques.

Qu'on me permette de faire une dernière remarque à propos de la connaissance historique comme synthèse. On pourrait croire que, lorsque je parle d'une synthèse, j'envisage la subsomption d'une collection complexe de détails sous des concepts très généraux de quelque espèce - par exemple, le concept de " déclin culturel ", ou celui de "triomphe commercial ". Et vraiment, les réalisations synoptiques des historiens peuvent, jusqu'à un certain point, être conçues de cette manière. Une foule d'actions et d'événements, conçus d'abord comme discontinus, se trouvent finalement perçus comme constituant quelque chose de collectif. C'est l'ensemble comme tel que l'historien explique.

On aura tort cependant de considérer une synopsis historique comme étant seulement une espèce d'explication par classification. Car les totalités que les récits historiques dessinent débordent typiquement les concepts qu'emploient les historiens pour les désigner. Et ce qui les intéresse le plus, c'est précisément ce débordement. On pourrait être certain, par exemple, que, par une expression synoptique comme "La Révolution française ", ils ne veulent pas seulement indiquer une myriade de choses qu'on peut qualifier collectivement de "révolutionnaires" et de "françaises". Ce qui serait en vue, même quand l'historien se sert de termes généraux appartenant à la description ordinaire des faits sociaux, c'est une configuration d'actions et d'événements qui pourrait être unique. La conscience de l'unicité de ce qu'il voit tout ensemble pousse quelquefois l'historien à abandonner le langage ordinaire des faits sociaux et à employer des métaphores comme celle de "la renaissance ». De plus, ce que l'historien voit tout ensemble pourrait être saisi comme tel, à un certain niveau, sans être subsumé du tout sous des concepts généraux, pas même des concepts métaphoriques. Comme Mink l'a souligné, il arrive aussi souvent aux historiens d'exposer, que de caractériser, ce que leurs jugements synoptiques ont accompli, de sorte que les vraies conclusions de leurs enquêtes sont - peut-on dire - "inséparables " des oeuvres qui les contiennent." Encore une fois, bien que d'une façon assez différente de l'analyse de Donagan, la théorie philosophique touche ici à une conception traditionnelle de la nature de la recherche historique et de son sujet.

21. L.O. Mink, "The Autonomy of Historical Understanding ", History and Theory, V, 1965, et dans W.H. Dray (éditeur), Pbilosophical Analysis and History. 181. 


\section{V}

En conclusion, qu'il me soit permis de faire un petit inventaire. J'ai examiné trois aspects de la connaissance historique qui me paraissent importants pour toute théorie philosophique de l'histoire, et qui me semblent ou bien incompatibles avec le point de vue de Hempel, ou bien obscurcis par lui de quelque manière. ${ }^{22}$ Ces aspects sont : son intérêt à l'idiosyncrasie individuelle; sa relativité aux points de vue des historiens ; et sa rétrospectivité, liée aux jugements synoptiques. Au cours de ma discussion, $j$ 'ai distingué six sortes d'explication en histoire qui, pour une raison ou une autre, s'éloignent du modèle hempelien. Ce sont les explications de Donagan en termes de dispositions individuelles ; les explications par une hypothèse de pensée ; les explications causales en termes de ce qui fait être ; les explications en termes de ce qui permet d'être ; les explications qui subsument une foule de choses sous des concepts généraux ; et celles qui relient des détails à de grandes configurations - les explications "non séparables" de Mink. En essayant de clarifier un peu ces six modèles explicatifs, j’ai souligné certains points précis où s'élève, non seulement la question du relativisme, mais aussi celle de l'indéterminisme.

Pour finalement revenir à mon point de départ, puis-je ajouter que tout cela, surtout en ce qui concerne l'explication en histoire, risque de ne pas être reconnu si on commence a priori avec un concept semblable à celui de Hempel. Ce qui me gêne, en fin de compte, dans la théorie hempelienne, c'est son peu de pertinence à l'égard d'une grande partie du travail des historiens. Son défaut principal, il me semble, est de manquer d'" empathie " avec la pratique. Et dans l'analyse philosophique d'une discipline ou d'un type de savoir, la logique, privée d'une telle "empathie ", est inutile. La première tâche d'une analyse est d'être correcte en ce qui concerne les concepts fondamentaux du domaine en question. Mais c'est exactement sur ce plan que l'approche hempelienne échoue.

\section{Université d'Ottawa}

22. J'ai discuté plus à fond quelques-uns de ces thèmes dans les articles suivants : “ Singular Hypotheticals and Historical Explanation ", dans L. Gross (éditeur), Sociological Theory: Inquiries und Paradigms, Harper and Row, 1967; "On Importance in History ", dans H.E. Kiefer et M.K. Munit\% (éditeurs), Mind, Science and History, N.Y.U. Press, 1970; "On the Nature and Role of Narrative in Historiography ",History and Theory, X, 1971, 153-171. 